\title{
A CASE REPORT: ANCA-NEGATIVE GRANULOMATOSIS WITH POLYANGIITIS PRESENTING AS AN INTRACRANIAL TUMOR SPREADING FROM THE NASAL CAVITY, AND MULTIPLE BRAIN INFARCTIONS.
}

\author{
Reetta Kivioja', Tom Pettersson², Dan Nordström², Antti Markkola³, Pekka Virtanen³, Anders Paetau, \\ Jukka Putaala ${ }^{1}$
}

1) Clinical Neurosciences, University of Helsinki and Department of Neurology, Helsinki University Hospital, Finland

2) Department of Medicine, Helsinki University Hospital and Clinicum, University of Helsinki, Finland

3) HUS Medical Imaging Center, Meilahti hospital and Department of Radiology, Helsinki University Hospital, Finland

4) Department of Pathology, HUSLAB and Helsinki University Hospital, Finland

A 76-year-old Caucasian woman was referred to neurological emergency unit with a history of four transient aphasia attacks. A saddle nose appearance and severe hypertension (230/95 $\mathrm{mmHg}$ ) were noted. Brain computer tomography revealed a white matter hypodensity in the base of the left frontal brain lobe. Magnetic resonance imaging showed chronic paranasal sinus inflammation, which had spread through the skull base into the anterior cranial fossa (Figure 1A,B).

Later on the patient developed severe headache, slight dysarthria, episodic vertigo with diplopia, intermediate cognitive impairment, and a resting tremor. Vast serum and cerebrospinal fluid examinations taken at 1 and 4 months were unremarkable, including negative ANCA-antibodies.

A stereotactic brain biopsy was performed at four months revealing a lymphocyte-rich perivascular small vessel inflammation invading the vessels walls both in cortex and in adjacent gyral white matter (Figure 2A).

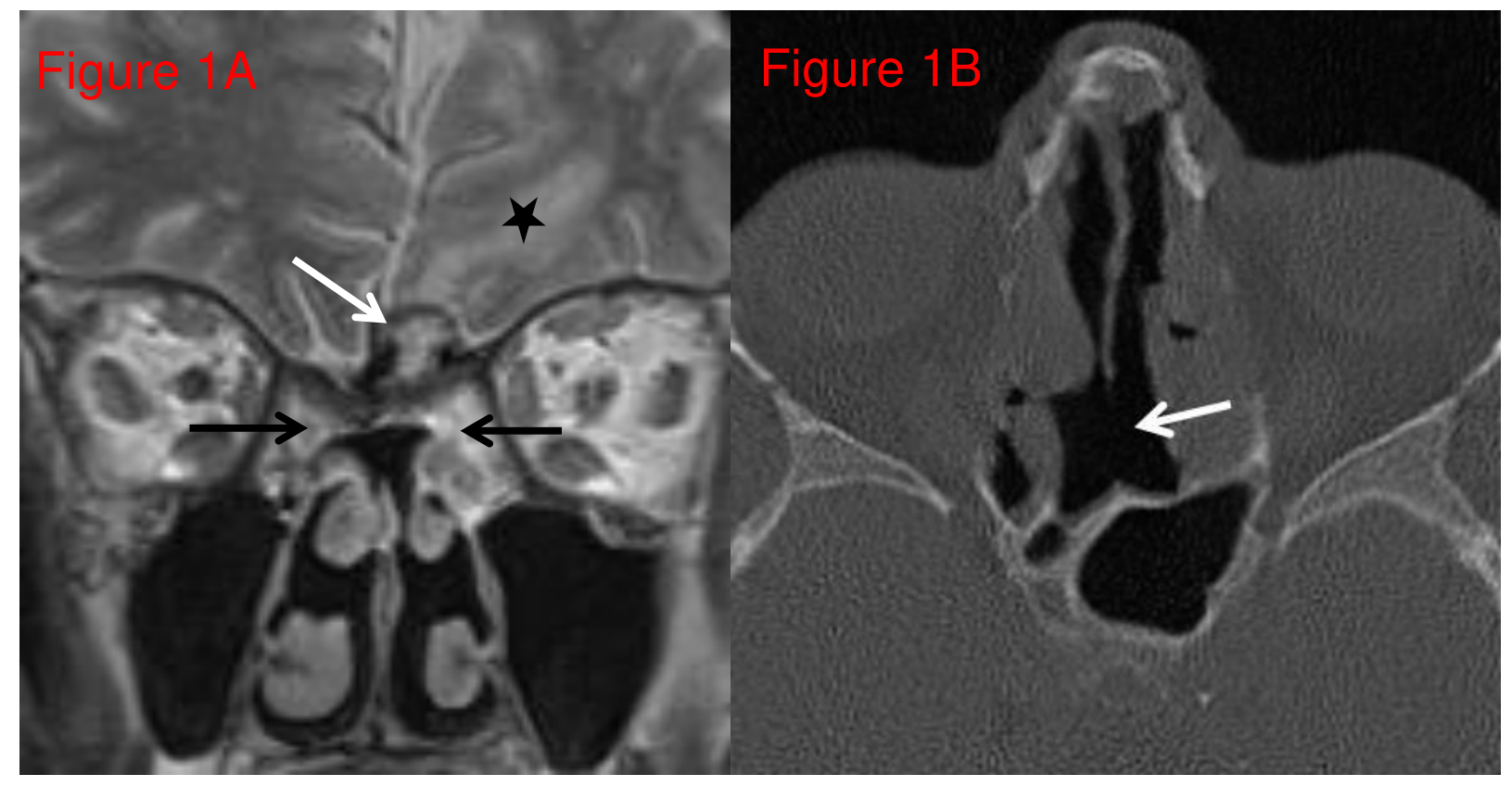

The findings were interpreted representing a small vessel vasculitis with no necrotizing or granulomatous features. A control brain MRangiography (MRA) and MRI showed arterial caliber variation compatible with intracranial arteritis (Figure 2B and multiple acute brain Infarctions (Figure 2C,D).

High-dose intravenous methylprednisolone (1.5 g x 1) treatment was immediately commenced followed by oral prednisolone (60 $\mathrm{mg}$ daily) and three intravenous cyclophosphamide cycles (1000 mg every two weeks). Cyclophosphamide was continued orally at a dose of $2 \mathrm{mg} / \mathrm{kg}$ daily for two months. Three months later a clear radiological and clinical improvement was noted.

The clinical picture combined with the radiological findings and histology of small vessel vasculitis in CNS, was found to be best compatible with moderately certain ANCA-negative granulomatosis with polyangiitis (GPA).

Figure 1A: Inflammatory process (black arrows) spreading through the ethmoidal fovea into the anterior cranial fossa and resulting in a small epidural inflammatory lesion (white arrow) with reactive brain edema (black star).

Figure 1B: Posterior septum perforation (arrow) typical for GPA.

Figure 2A: Stereotactic brain biopsy. In the center is seen a small vessel surrounded by a quite intense lymphocyte-rich perivascular inflammation invading also the vessel wall.

Figure 2B: Moderate arterial caliber variation in posterior cerebral arteries and lesser changes in medial cerebral and pericallosal arteries. Figure 2C: DWI MRI of the brain shows multiple recent infarctions. Figure 2D: FLAIR MR image of the brain shows multiple deep basal ganglia, subcortical and cortical infarctions.

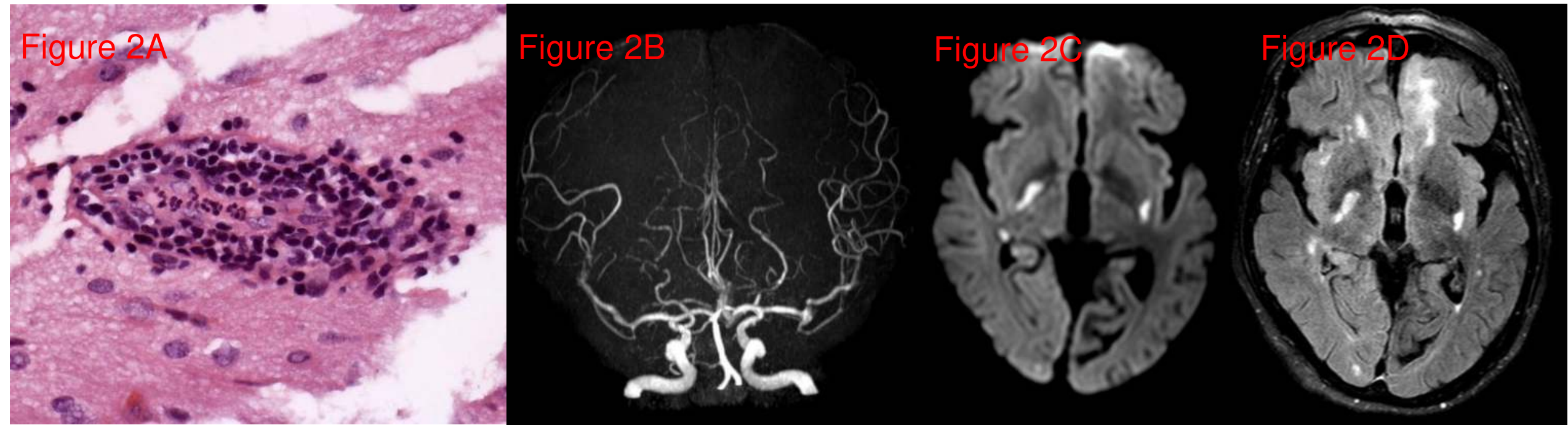

